\title{
Self-injurious behaviour in young children with Lesch-Nyhan Syndrome.
}

\author{
Hall, S., Oliver, C. \& Murphy, G. \\ Cerebra Centre for Neurodevelopmental Disorders, \\ School of Psychology, \\ University of Birmingham
}

Please use this reference when citing this work:

Hall, S., Oliver, C. \& Murphy, G. (2001). Self-injurious behaviour in young children with Lesch-Nyhan Syndrome. Developmental Medicine and Child Neurology, 43, 745-749.

The Cerebra Centre for Neurodevelopmental Disorders,

School of Psychology, University of Birmingham, Edgbaston, Birmingham, B15 2TT

Website: www.cndd.Bham.ac.uk

E-mail: cndd-enquiries@contacts.bham.ac.uk 


\title{
Self-injurious Behaviour in Young Children with Lesch-Nyhan Syndrome
}

\author{
Scott Hall* PhD, \\ School of Psychology, University of Birmingham, Birmingham, UK \\ Chris Oliver, $\mathrm{PhD}$, \\ School of Psychology, University of Birmingham; Birmingham, UK.
}

Glyn Murphy, PhD,

Tizard Centre, University of Kent, Canterbury, UK

*Correspondence to

School of Psychology

University of Birmingham

Edgbaston

Birmingham

B15 2TT, UK.

Fax: 01214149897

e-mail : s.s.hall@bham.ac.uk 


\begin{abstract}
The early development of self-injurious behaviour (SIB) in three young boys with LeschNyhan syndrome was examined by means of parental interviews and by direct observations completed at three to four monthly intervals over an 18-month period. The results suggested that the self-injury began in a different way from that of other young children with autism and/or developmental disabilities in that, from the start, self-injurious responses were sudden and violent, rather than emerging gradually over time. Drastic measures, such as removal of the teeth or provision of tooth guards, were often taken to prevent further tissue damage. Direct observations showed that the boys had lower rates of self-injury than other children with autism and/or severe developmental disabilities who had recently stared to self-injure, but their carers were highly concerned about the behaviour. Sequential analysis of the observational data indicated that on some occasions the boys were more likely to self-injure during periods of low social interaction, suggesting that their self-injury may have been influenced by environmental factors. The theoretical and practical implications of these findings are discussed.
\end{abstract}

Running Head: Lesch-Nyhan syndrome 


\section{Self-injurious behaviour in \\ Young Children with Lesch-Nyhan Syndrome}

Lesch-Nyhan Syndrome involves an inborn error of purine metabolism, due to the absence (or very low levels) of hypoxanthine-guanine phosphoribosyltransferase (HPRT) enzyme (Lesch \& Nyhan, 1964). The disorder has an X-linked pattern of inheritance, occurring in 1 in 800,000 (Harris, 1992), and is often diagnosed late, frequently being thought to be athetoid cerebral palsy (Christie et al., 1982). A characteristic feature of Lesch-Nyhan syndrome is the appearance of intractable self-injurious behaviour, usually in the form of severe lip and finger biting or chewing that often requires extreme management techniques such as the application of restraints and/or extraction of teeth at an early age. Whilst the syndrome is characterised by high levels of uric acid (Lesch \& Nyhan, 1964), reduction of these levels does not appear to affect the manifestation of self-injury (Christie et al., 1982).

It has long been proposed that for individuals with Lesch-Nyhan syndrome, there appear to be biological determinants to the self-injurious behaviour that are not often seen in other individuals with severe self-injury (Nyhan, 1994). This hypothesis has arisen from three main observations. Firstly, all individuals with the syndrome show the behaviour, which appears to start in the third year of life on average and then continues inexorably thereafter (Christie et al., 1982). Secondly, the topographies seem more predictable in Lesch-Nyhan syndrome, in that lip and cheek biting almost always occur in the early years, and other behaviours, such as trapping fingers in door hinges and wheel-spokes of wheelchairs, often appear later. Thirdly, the boys and young men seem to welcome protective restraining devices and appear to become extremely agitated when these are removed (Christie et al., 1982; Anderson \& Ernst, 1994). 
Conflicting evidence concerning the biological nature of SIB in Lesch-Nyhan syndrome comes from a number of studies that have documented the influence of social and environmental factors in the maintenance of SIB (Duker, 1975; Anderson et al., 1978; Bull \& LaVecchio, 1978; Gilbert et al., 1979; Wurtele et al., 1984). Anderson et al., (1978) found that SIB increased in one child when it was followed by social attention, and behavioural treatments that involved a re-arrangement of environmental contingencies (e.g., extinction, DRO, and extinction plus DRO) reduced rates of SIB in five boys, at least in the short term. Duker (1975) also reported that ignoring one boy's self-biting resulted in a decrease in SIB, suggesting that the boy's SIB may, in the past, have been reinforced by social attention. Interestingly, in studies conducted by Anderson et al., $(1977,1978)$ punishment by contingent shock appeared to be completely ineffective in reducing rates of SIB, suggesting that individuals with Lesch-Nyhan syndrome are unable to learn to avoid aversive stimulation.

Pharmacological treatments for SIB in Lesch-Nyhan syndrome have failed to produce any lasting effect on rates of SIB. Trials with 5-HTP (a serotonin agonist), fluoxetine (a serotonin reuptake inhibitor), naltrexone (an opiate antagonist) and fluphenazine (a dopamine antagonist) have shown either no effect or produced reductions in SIB for only short periods of time (Nyhan et al., 1980; Anderson et al., 1976; Nyhan, 1994). Interestingly, Goldstein et al., (1985) found fluphenazine completely eliminated SIB in one boy aged 20 months (whose SIB had just started) whereas in another child, aged 15, fluphenazine actually increased levels of SIB. More studies of treatments that are begun early in the child's life are clearly needed.

In recent years, a number of sophisticated improvements to both the behavioural analysis and treatment of self-injury in individuals with autism and/or developmental disabilities have occurred (Carr \& Durand, 1985; Durand \& Carr, 1991; Iwata et al., 1994; Murphy et al., 1993; Wacker et al., 1990). Studies have shown that SIB can be maintained by environmental consequences such as the contingent removal of demands, the contingent 
provision of attention and/or tangible items such as toys and by automatically produced sensory consequences (Carr, 1977). Far less attention has been paid to the origins of SIB i.e. the first emergence of self-injury in the child's repertoire (Murphy \& Wilson, 1985, p. 90; Guess \& Carr, 1991). Such research is important, given that it could inform early intervention programmes and promote the early detection of SIB, before it becomes established.

In a previous study, the authors tracked sixteen children with autism and/or developmental disabilities who had recently started to show SIB (Murphy et al., 1999; Hall et al., 2001). Observations of the children were conducted at the child's school every 3 months over a two-year period and associations between environmental events and early SIB were assessed. In four of the children whose SIB had increased, low levels of social interaction appeared to be significantly associated to the SIB, suggesting that elements of the social environment were responsible for its exacerbation. Apart from this study, there have been extremely few studies of the early development of self-injurious behaviour in young children. Yet, if self-injury in boys with Lesch-Nyhan syndrome develops earlier than SIB seen in other children and if it is somewhat different in nature, it is important to describe these differences. In addition, if self-injury can be shown to be associated to environmental factors, this would have implications for the management and early intervention of SIB in Lesch-Nyhan syndrome and could suggest that there may be an interaction between biological and environmental factors in the determinants of the behaviour.

In this research, very young children with Lesch-Nyhan syndrome were studied over a period of 18 months, to examine the emergence of self-injurious behaviour and to examine the factors associated with its origins. In addition to interviews conducted with the children's parents, direct observations of the children were conducted at regular intervals in order to determine whether the children's early SIB was associated with environmental events. 


\section{Method}

\section{$\underline{\text { Participants }}$}

Boys with Lesch-Nyhan syndrome who were under three years of age were selected for the study, regardless of whether they had begun to show self-injurious behaviours. Five families were contacted through paediatric neurologists in the south east of England and through the purine screening laboratory at two London hospitals, through which all of the boys had their diagnoses confirmed. Families were sent a consent letter describing the study and were asked if they would like to participate. It was made clear to all the children's parents that participation in the study would not deny the child access to local treatment services (if required) during the study and that feedback would be given at the end of the study. Three of the families agreed to participate.

George was 17 months old at the start of the study and attained an age equivalent score on the Vineland Adaptive Behavior Scales (Sparrow, Balla \& Chiccetti, 1984) of 9 months. George's parents had noticed 'orange sand' in their son's nappies in the first week and noted his poor head control at 3 months. Referral to the local paediatrician led to a diagnosis at 10 months (by a London teaching hospital). George's parents reported that he had scratched his eye with his fingernails at 11 months, pulled his own hair a few times at 14 months and had bit his fingers 3 times over the last 3 months. His mother felt that none of these behaviours were "true SIB" but were "accidental" and probably a function of George's poor motor control. It was also reported that George would bang his head on the floor as a result of reflex extension when sitting, approximately once per month. George's parents would say "no" when the behaviour occurred and then give him a cuddle. If George's hands went up to his mouth, he was told to put them down.

Adam was 30 months at the start of the study and attained an age equivalent score on the Vineland of 6 months. He had an early history of projectile vomiting but went home after 
his birth until the worsening of his vomiting led him to return to hospital at 3 weeks of age. He continued to vomit, became dehydrated and was transferred to a London teaching hospital where he was diagnosed at 5 weeks of age. Adam's mother reported that he had started biting his bottom lip at 21 months when he was in hospital (for constipation). This escalated on return home and occurred in bursts in the evening, especially if Adam was ill or if he was in discomfort at night (e.g. in an uncomfortable position in his cot). At the beginning of his involvement in the study, a gum shield was being made for him to wear at night. If Adam's mother was present when the behaviour occurred, she reported that she would usually say "no" and pull his bottom lip away from his teeth.

Peter was 25 months at the start of the study and attained an age equivalent score on the Vineland of 8 months. Referral to the local paediatrician (by his GP) finally led to a diagnosis at 14 months by a neurologist in a Regional teaching hospital. Peter's parents reported that he had bitten the inside of his cheek at 15 months and that this had occurred during the night. He had then bitten his lower lip (producing bleeding) when out shopping at 18 months. At the time of his involvement in the study, it was reported that Peter engaged in occasional 'accidental' hair pulling and face scratching (due to poor motor control). Peter would also engage in lip biting when he was in discomfort or in order to "say no". Biting the inside of his cheek occurred during the night, about once per week. Peter's parents reported that gum shields had been tried but had not been effective. Peter had some teeth removed to prevent the biting (but new teeth were growing in). When lip biting occurred, Peter's parents reported that they would usually put their fingers in his mouth.

\section{$\underline{\text { Measures }}$}


In observations, child responses recorded included body banging, head banging, body hitting, head hitting, eye poking, eye pressing, hand mouthing, hand biting, scratching or rubbing of the skin and hair pulling. Operational definitions for each of these responses can be found in Murphy et al. (1999). Two teacher behaviours were also recorded. These were: demands, defined as any verbal or physical direction by the teacher in order for the child to complete an action or task, and attention, defined as any other physical or verbal contact made by the teacher to the child e.g., touching, response blocking, offering drinks or food, reprimanding and commenting. At analysis, the computer automatically coded three additional codes. These were: demand removal, defined as the discontinuation of demands for 10-s following the occurrence of a demand (or the time between successive occurrences of demands, if demands recurred within 10-s); attention removal, defined as the discontinuation of attention for 10-s following the occurrence of attention (or the time between successive occurrences of attention, if attention recurred within 10-s) and no interaction, defined as the absence of demands, attention, demand removal and attention removal.

All responses were recorded on a laptop computer (Olivetti Quaderno, Model PT-XT-20) using software that allowed continuous documentation of the frequency and/or duration of each behaviour and their interrelations (see Repp et al., 1989). The reliability of this coding system has been reported elsewhere (see Murphy et al., 1999).

At each observation point, nursery teachers were also asked to indicate how 'concerned' they were about the child's SIB by rating the topographies of SIB (listed above) on a 5-point scale with 0 indicating "no concern", 1 indicating "weak concern", 2 indicating "definite concern", 3 indicating "strong concern" and 4 indicating "extreme concern". 


\section{Procedure}

All children were observed in their regular nursery school at the beginning of the study and at 3-monthly intervals over an 18-month period. Nurseries usually contained the child's nursery teacher, one or more teacher assistants and at least 4 but no more than 10 other children. Each child in the study was observed for a 3 to 4 hour period at each observation point. Throughout each observation period, the observer followed the child as unobtrusively as possible and did not interact with the child at any time. Teachers were reminded prior to each observation period to ignore the presence of the observer and to interact normally with the child. Observations included a representative sample of activities: meals, group activities, individual work, and leisure time. The observer stood in the corner of the room and out of the child's line of sight.

The aim of the study was to conduct direct observations in nurseries for each child, once every three months for 18 months. However, the mean length of interval between follow-ups for each child was actually 4 months (range 3 months to 7 months). A long follow-up interval (i.e., 7 months) occurred when Peter was admitted to hospital for a treatment unrelated to SIB. The mean total length of follow-up period for the boys with Lesch-Nyhan syndrome was 17.33 months (range 16 to 18 months).

Association between SIB and environmental events. In order to examine the association between a child's early SIB and socially mediated environmental events, the conditional probability of each teacher response, $\underline{T}_{i}$, given SIB was calculated; $\underline{p}\left(\underline{T}_{i} \mid S I B\right)$. This was done by imposing 10 -s intervals on the data and determining the number of times that a particular teacher response occurred given that SIB had occurred in each 10-s interval and dividing this number by the number of 10 -s intervals during which SIB occurred. However, since the base rate of SIB was low at each observation point however, it was possible that high conditional 
probabilities could occur simply by chance. To control for this possibility, the conditional probability of SIB given the occurrence of a particular teacher response, $\underline{T}_{i}$, was also calculated at each observation point for each child; $\mathrm{p}\left(\mathrm{SIB} \mid \underline{\mathrm{T}}_{\mathrm{i}}\right)$. This was done by determining the number of times that SIB had occurred given that a particular teacher response had occurred in each 10 -s interval and dividing this number by the number of 10 -s intervals during which the particular teacher response occurred.

The information in these two conditional probabilities can be combined using the $\underline{Z}$ statistic (Bakeman \& Gottman, 1997; Bakeman \& Quera, 1995). The formula for $\underline{Z}$ is given as follows:

$z_{i j}=\left(x_{i j}-m_{i j}\right) / \sqrt{m_{i j}\left(1-p_{i}\right)\left(1-p_{j}\right)}$

where $\underline{x}_{i j}=$ observed joint frequency of SIB and $\underline{T}_{i}, \underline{m}_{i j}=$ expected joint frequency of SIB and $\underline{T}_{i}, \underline{p}_{i}=$ unconditional probability of SIB, and $\underline{p}_{j}=$ unconditional probability of $\underline{T}_{i}$. A high value for $\underline{Z}$ indicates a significant association between SIB and the teacher response (i.e., higher than would be expected by chance). All conditional probabilities and $\underline{Z}$ statistics were calculated using the SDIS-GSEQ software (Bakeman \& Quera, 1995). Only the $\underline{Z}$ statistics will be reported here.

Results

The upper panel of Figure 1 shows the percentage of 10-s intervals during which SIB occurred at each observation point for each of the boys. 
The figure shows that SIB occurred for less than $10 \%$ of the time in all observations. The topographies observed for the three boys (also identified by the children's teachers) were selfscratching and hitting head on objects for George, self-biting, self-scratching, and hitting head on objects for Adam and self-scratching, hitting head on objects and self-pinching for Peter.

George's teacher had indicated that her concern about George hitting his head on objects was 'definite' but that her concern was 'weak' for his self-scratching. Adam's teacher had indicated that her concern about his self-biting and hitting his head on objects was 'definite' and that her concern about his self-scratching was 'strong'. She also indicated at the last observation that Adam had begun to rub his body against the straps of his chair producing sores and had been hitting an open wound against his chair. Peter's teacher had indicated that her concern for his self-injurious behaviours was 'weak'.

The middle panel of Figure 1 shows the percentage of 10-s intervals during which the various teacher behaviours (i.e., attention, demands, attention removal, demand removal and no interaction) occurred at each observation point. The figure shows that Adam received high levels of attention throughout the duration of the study and was rarely left alone. In contrast, George and Peter were left alone fairly often, although Peter also received high levels of demands at the third and fifth observation points.

The lower panel of Figure 1 shows the corresponding $\underline{Z}$-score statistics, indexing the extent to which each child's SIB was associated with each teacher behaviour at each observation point over and above what would be expected by chance ${ }^{1}$. The figure shows that for 5 out of the 6 observation points for George, for 4 of 5 observation points for Adam and

\footnotetext{
${ }^{1}$ For Peter's last observation session, the number of intervals containing SIB was less than 10 . Z-scores for this observation should therefore be interpreted with caution (see Bakeman \& Gottman, 1997, p. 145).
} 
for 2 of the 5 observation points for Peter, there was a positive association between SIB and 'no interaction'. These data indicate that, on occasions, SIB was more likely to occur during periods when the children were left alone. These data suggest that SIB shown by children with Lesch-Nyhan may have been influenced by environmental factors, in this case, low levels of social interaction.

\section{Discussion}

Several investigators have commented on the severity and intractability of SIB seen in individuals with Lesch-Nyhan syndrome (e.g., Dizmang \& Cheatham, 1970; Christie et al., 1982; Anderson \& Ernst, 1994). All three of the boys with Lesch-Nyhan syndrome in the present study showed lip and/or finger biting before the age of three years. Despite their young age and the relatively low rate of SIB seen in the children, the self-injury was intense when it occurred and two of the boys were already requiring protective devices in the mouth and/or tooth removal to prevent lip and/or cheek biting. In addition, SIB sometimes appeared at night, for no clear reasons. These data support the findings of other studies and suggest that SIB in Lesch-Nyhan syndrome may indeed be an inevitable and intractable feature of the disorder.

The data in the present study contrast to similar data collected by the authors on sixteen children with autism and/or severe developmental disabilities (see Hall et al., 2001; Murphy et al., 1999). Here, SIB appeared much later, was far slower to develop, a variety of topographies were shown, and initial responses were far more insidious, with only one of the children requiring restraining or protective devices nearly two years after SIB had started. It appears therefore that SIB seen in Lesch-Nyhan syndrome is qualitatively different to that 
seen in other individuals without the syndrome and is more severe and difficult to manage, even in the early years.

Sequential analysis of the observational data in the present study revealed that on several occasions, the self-injury shown by all three boys appeared to be influenced by environmental factors. Specifically, SIB was more likely to occur when the children had been left alone without social contact. It seems likely that deprivation of social interaction in the classroom may have served to evoke the SIB which subsequently may have led to an increase in the level of social interaction (see Oliver, 1993). Interviews with the parents of all three children indicated that they responded to the SIB either by saying "no" or giving the children a cuddle, suggesting that the behaviours may have been maintained by access to social contact. These data provide support for the experimental evidence obtained by Anderson and his colleagues who showed that SIB in Lesch-Nyhan could be reinforced by access to contingent attention.

Given these findings, it would appear that behavioural intervention strategies, aimed at reducing the occurrence of self-injury as soon as it appears, could be appropriate for children diagnosed with Lesch-Nyhan syndrome. One such early intervention strategy could be to actively promote early communicative behaviours (e.g., signing or verbal mands) in the children's repertoires at a very early stage (Carr \& Durand, 1985; Durand \& Carr, 1991). Another behavioural intervention for SIB could be to differentially reinforce non-SIB behaviours (DRO) (c.f. Anderson et al., 1978). Before these interventions are implemented, a functional analysis should be conducted in order to determine which reinforcers (if any) are currently maintaining the child's SIB (Iwata et al., 1994).

It is only recently that the interactions between biological and developmental systems and the environment have become the focus of research (Guess \& Carr, 1991; Harris, 1995; Oliver, 1993; Schroeder et al., 1990). The fact that environmental factors may also contribute 
to the development of SIB in children with Lesch-Nyhan syndrome suggests that there may be an interaction between biological and environmental factors in the development of SIB in Lesch-Nyhan syndrome. Whilst it is hoped that a pharmacological treatment for SIB in LeschNyhan syndrome could appear soon, the potential role of environmental factors on SIB should not be forgotten. 


\section{References}

Anderson LT, Herrman L. \& Dancis, J. (1976) The effect of L-5-hydroxytryptophan on selfmutilation in Lesch-Nyhan disease: A negative report. Neuropaediatrie, 7; 439-442.

Anderson LT, Dancis L, \& Alpert, M. (1978) Behavioral contingencies and self-mutilation in Lesch-Nyhan disease. Journal of Consulting and Clinical Psychology 46; 529-536.

Anderson LT, Dancis J, Alpert M, Huermann L. (1977) Punishment learning and selfmutilation in Lesch-Nyhan disease. Nature 265; 461-463.

Anderson LT, Ernst M. (1994) Self-injury in Lesch-Nyhan disease. Journal of Autism and Developmental Disorders 24; 67-81.

Bakeman R, Gottman JM. (1997) Observing Interaction: An Introduction to Sequential

Analysis. $2^{\text {nd }}$ Edition. Cambridge: Cambridge University Press.

Bakeman R, Quera V. (1995) Analyzing Interaction: Sequential Analysis with SDIS and

GSEQ. Cambridge, Cambridge University Press.

Bull M, LaVecchio F. (1978) Behaviour therapy for a child with Lesch-Nyhan syndrome. Developmental Medicine and Child Neurology 20; 368-375.

Carr EG. (1977) The motivation of self-injurious behaviour: a review of some hypotheses. Psychological Bulletin 84; 800-816. 
Carr EG, Durand VM. (1985) Reducing behaviour problems through functional communication training. Journal of Applied Behaviour Analysis 18; 111-126.

Christie R, Bay C, Kaufman IA, Bakay B, Borden M, Nyhan WL. (1982)

Lesch-Nyhan Disease: clinical experience with nineteen patients. Developmental Medicine \&

Child Neurology 24; 293-306.

Dizmang LH, Cheatham CF. (1970) The Lesch-Nyhan syndrome. American Journal of Psychiatry $127 ; 671-677$.

Duker P. (1975) Behavioural control of self-biting in a Lesch-Nyhan patient. Journal of Mental Deficiency Research 19; 11-19.

Durand VM, Carr EG. (1991) Functional communication training to reduce challenging behaviour: maintenance and application in new settings. Journal of Applied Behaviour Analysis $24 ; 251-264$.

Gilbert S, Spellacy E, Watts RWE. (1979) Problems in the behavioural treatment of selfinjury in the Lesch-Nyhan syndrome. Developmental Medicine \& Child Neurology 21; 795800.

Goldstein M, Anderson LT, Reubin R. \& Dancis J. (1985) Self-mutilation in Lesch-Nyhan disease is caused by dopaminergic denervation. Lancet, 1, 338-339. 
Guess D, Carr, EG. (1991) Emergence and maintenance of stereotypy and self-injury. American Journal on Mental Retardation 96; 299-319,

Hall S, Oliver C, Murphy G. (2001) The early development of self-injurious behavior: An empirical study. American Journal on Mental Retardation.

Harris J. (1992) Neurobiological factors in self-injurious behaviour. In: Luiselli JK, Matson JL, Singh N, editors. Self-injurious Behaviour: Analysis Assessment and Treatment. New York: Springer-Verlag. p 59-92.

Harris, J. (1995) Genetic metabolic disorders associated with behavioural phenotypes: LeschNyhan Disease. In: Developmental Neuropsychiatry vol. 2. New York: Oxford University Press. p 306-319.

Iwata BA, Pace GM, Dorsey MF, Zarcone JR, Vollmer TR, Smith RG, Rodgers TA, Lerman DC, Shore BA, Mazeleski JL et al. (1994) The functions of self-injurious behavior: An experimental epidemiological analysis. Journal of Applied Behavior Analysis 27; 215-240.

Lesch M, Nyhan WL. (1964) A familial disorder of uric acid metabolism and central nervous system function. American Journal of Medicine 36; 561-570.

Murphy G, Hall S, Oliver C, Kissi-Debra R. (1999) Identification of early self-injurious behaviour in young children with intellectual disability. Journal of Intellectual Disability

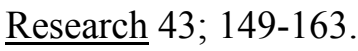


Murphy G, Oliver C, Corbett J, Crayton L, Hales J, Head D, Hall S. (1993)

Epidemiology of self-injury, characteristics of people with severe self-injury and initial

treatment outcome. In: Kiernan C, editor. Research to Practice? Implications of research on

the challenging behaviour of people with learning disability. Kidderminster ; BILD

Publications. p 1-35.

Murphy G, Wilson, B. (1985) Self-injurious behaviour. Kidderminster; BIMH Publications.

Nyhan WL, Johnson HG, Kaufman IA. \& Jones KL (1980) Serotonergic approaches to the modification of behavior in the Lesch Nyhan syndrome. Applied Research in Mental

Retardation, 1, 25-40.

Nyhan WL (1994) The Lesch-Nyhan disease. In: Thompson T, Gray DB, editors. Destructive behavior in developmental disabilities: Diagnosis and treatment. Thousand Oaks, CA: Sage Publications. p 181-197.

Oliver C. (1993) Self-injurious behaviour: From response to strategy. In: Kiernan C, editor. Research to Practice? Implications of research on the challenging behaviour of people with learning disability. Kidderminster; BILD Publications. p 135-188.

Oliver C, Murphy GH, Corbett JA. (1987) Self-injurious behaviour in people with mental handicap: a total population study. Journal of Mental Deficiency Research 31; 147-162.

Repp AC, Harman ML, Felce D, Van Acker R, Karsh KG. (1989) Conducting behavioural assessments on computer collected data. Behavioural Assessment 11; 249-268. 
Schroeder SR, Breese GR, Mueller RA. (1990) Dopaminergic mechanisms in self-injurious behaviour. Advances in Developmental and Behavioural Paediatrics 9; 181-198.

Sparrow SS, Balla DA, Chiccetti DV. (1984) Vineland Adaptive Behavior Scales: Interview Edition. Survey Form manual. Circle Pines, MN: American Guidance Service.

Wacker DP, Steege MW, Northup J, Sasso G, Berg W, Reimers T, Cooper L, Cigrand K, Donn L. (1990) A component analysis of functional communication training across three topographies of severe behaviour problems. Journal of Applied Behavior Analysis 23; 417 429.

Wurtele SK, King AC, Drabman RS. (1984) Treatment package to reduce SIB in a LeschNyhan patient. Journal of Mental Deficiency Research 28; 227-234.

\section{Acknowledgements}

We are very grateful to the children, their parents and teachers who helped in this study. This research was funded by the Mental Health Foundation, UK. 


\section{Figure Caption}

Figure 1. Percentage of 10-s intervals of SIB observed across follow-up observations for each child (upper panel), percentage of 10-s intervals of teacher behaviours observed (middle panel) and corresponding $\underline{Z}$-scores indexing the association between SIB and the teacher behaviours (lower panel). 
George

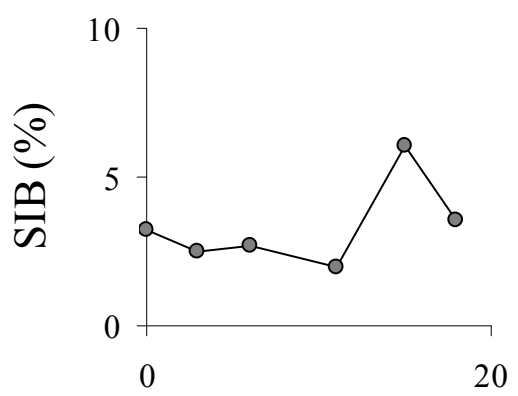

do

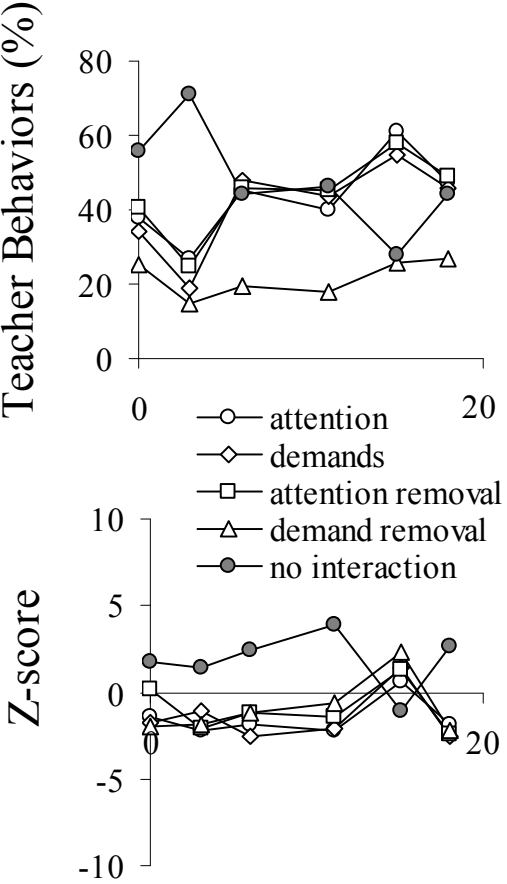

Adam
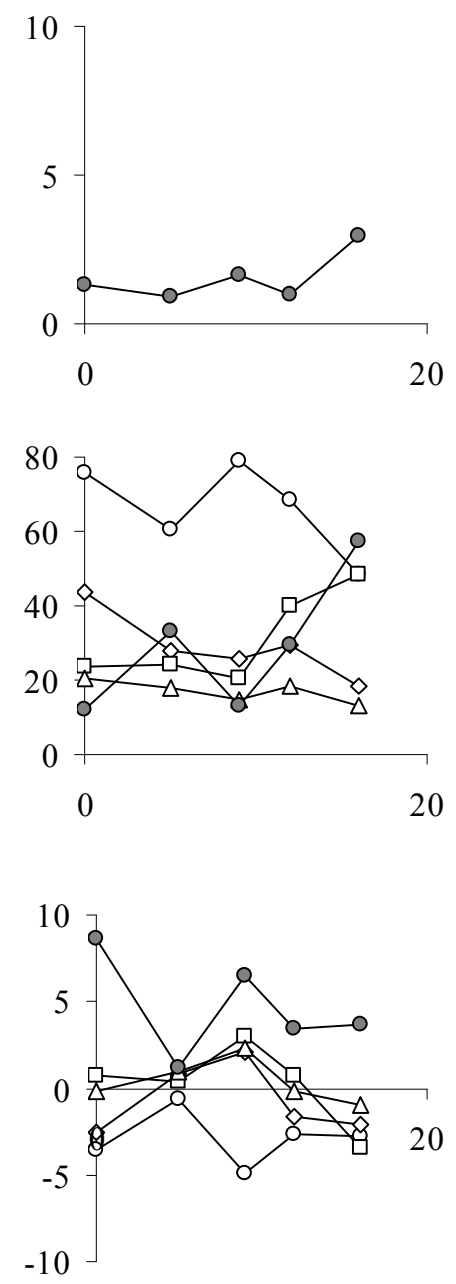

Peter
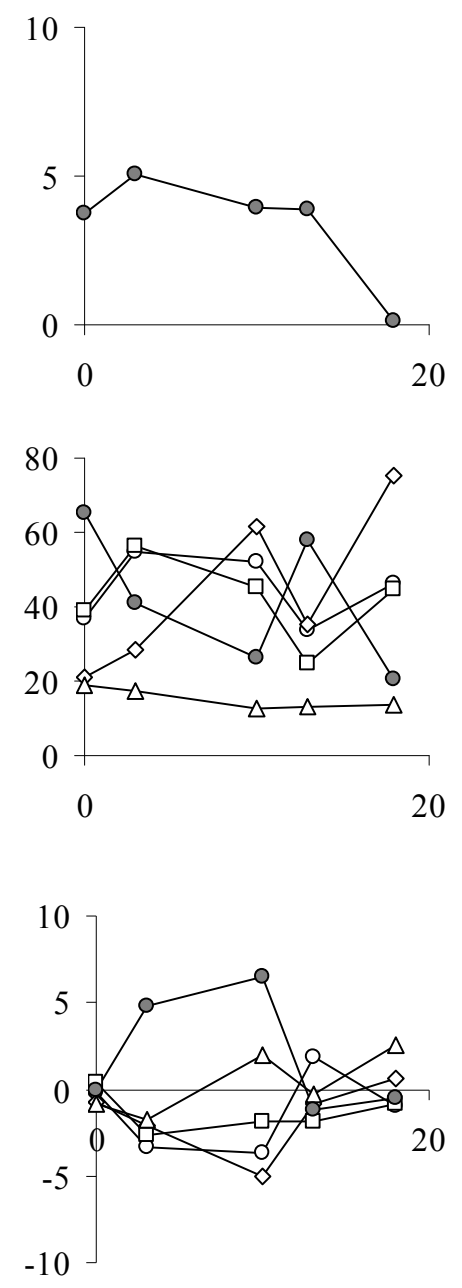

Time of Observation (months) 